\title{
Factors associated with mortality among the COVID-19 patients treated at Gulu Regional Referral Hospital: A retrospective study
}

\section{Steven Baguma}

Gulu Regional Referral Hospital

Christopher Okot

Gulu Regional Referral Hospital

Nelson Alema Onira

Gulu University, Faculty of Medicine, Department of Anatomy

Paska Apiyo

Gulu Regional Referral Hospital

Denis Acullu

Aga Kan Hospital, Mombasa, Kenya

\section{Paska Layet}

$$
\text { Lacor Hospital }
$$

Johnson Nyeko Oloya

Uganda Medical Association, Acholi Branch, Gulu, Uganda

Denis Ochula

Lamwo Local Goverment, Uganda

\section{Pamela Atim}

St. Joseph's Hospital, Kitgum, Uganda

\section{Patrick Odong Olwedo}

Amuru Local Government, Uganda

\section{Smart Godfrey Okot}

Kalongo Hospital, Agago, Uganda

\section{Freddy Wathum Drinkwater Oyat}

Uganda Medical Association, Acholi Branch

\section{Janet Oola}

Nwoya Local Government, Uganda

Judith Aloyo

Rhites-N, Acholi, Gulu City, Uganda

\section{Eric Nzirakaindi Ikoona}

ICAP at Columbia University, Sierra Leone

David Lagoro Kitara ( $\square$ klagoro2@gmail.com ) 
Gulu University, Faculty of Medicine, Department of Surgery https://orcid.org/0000-0001-7282-5026

\section{Research Article}

Keywords: COVID-19, Gulu Regional Referral Hospital, mortality, comorbidities, females, symptoms, signs

Posted Date: December 22nd, 2021

DOI: https://doi.org/10.21203/rs.3.rs-1191937/v1

License: @ (i) This work is licensed under a Creative Commons Attribution 4.0 International License. Read Full License

Version of Record: A version of this preprint was published at Frontiers in Public Health on April 11th, 2022. See the published version at https://doi.org/10.3389/fpubh.2022.841906. 


\section{Abstract}

Background: The advent of the novel coronavirus disease 2019 (COVID-19) has caused millions of deaths worldwide. There is a lack of data on the outcome of hospitalized African patients suffering from COVID-19.

This study aimed at identifying factors associated with hospital mortality in patients who suffered from COVID-19 at Gulu Regional Referral Hospital in Northern Uganda from March 2020 to October 2021.

Methods: This was a single-center, retrospective study in patients hospitalized with confirmed COVID-19 at Gulu Regional Referral Hospital in Northern Uganda. Socio-demographic characteristics, clinical presentations, comorbidities, duration of hospital stay, and treatment were analyzed, and factors associated with increased odds of mortality were determined.

Results: Of the 664 patients treated, $661(99.5 \%)$ were unvaccinated, $632(95.2 \%)$ recovered and 32(4.8\%) died. Mortality was highest in diabetics 11(34.4\%), cardiovascular diseases 12(37.5\%), hypertensive $10(31.3 \%)$, females $18(56.3 \%)$, $\geq$ 50-year-olds $19(59.4 \%)$, no formal education $14(43.8 \%)$, peasant farmers $12(37.5 \%)$ and those who presented with difficulty in breathing/shortness of breath and chest pain $32(100.0 \%)$, Oxygen saturation $\left(\mathrm{SpO}_{2}\right)<804(12.5 \%)$, general body aches and pains $31(96.9 \%)$, tiredness $30(93.8 \%)$ and loss of speech and movements 11(34.4\%). The independent factors associated with mortality among the COVID-19 patients were females AOR=0.220,95\% Cl:0.059-0.827; $p=0.030$; Diabetes mellitus AOR=9.014, 95\%Cl:1.726-47.067; $p=0.010$; tiredness AOR=0.059,95\% Cl:0.009-0.371; $p=0.0000$; general body aches and pains $A O R=0.066,95 \% \mathrm{Cl}: 0.007-0.605 ; p=0.020$; loss of speech and movement AOR $=0.134,95 \% \mathrm{Cl}: 0.270-0.660 ; p=0.010$ and other comorbidities $A O R=6.860,95 \% \mathrm{Cl}: 1.309$ $35.957 ; p=0.020$.

Conclusion: The overall hospital mortality was $4.8 \%$. Older age, people with diabetics, females, other comorbidities, severe forms of the disease, and those admitted to HDU were significant risk factors associated with hospital mortality. More efforts should be made to provide "Enhanced shielding"to the most vulnerable population to avoid preventable morbidity and mortality of COVID-19 in Northern Uganda.

\section{Introduction}

Coronavirus disease 2019 (COVID-19) caused by SARS-CoV-2 first emerged in Hubei Province, China in December 2019 [1]. Since then, not only has COVID-19 been considered a public health emergency of international concern, but it has been declared a global pandemic [1]. COVID-19 is an infectious disease caused by a new coronavirus that can be transmitted from one infected person to an average of 3 other people in a population [2]. This emerging disease is epidemiologically like severe acute respiratory syndrome (SARS) and the Middle East Respiratory Syndrome (MERS), which were previously epidemic [3]. Although most infected persons develop mild symptoms, some may develop respiratory failure, arrhythmia, shock, renal failure, cardiovascular injury, hepatic failure, and sometimes death $[4,5]$. 
At present, there is no effective antiviral treatment; only supportive care may be useful, for example, mechanical ventilation, extracorporeal membrane oxygenation (ECMO) to patients with refractory hypoxemia, or ECMO to patients with refractory hypoxemia [5]. The overall Case Fatality Rate (CFR) is $3.8 \%$ in COVID-19 worldwide [5]. CFR in patients with cardiovascular diseases, diabetes, hypertension, respiratory diseases, and cancers is estimated to be $13.2 \%, 9.2 \%, 8.4 \%, 8.0 \%$, and $7.6 \%$, respectively [6]. Several studies focusing on factors affecting the mortality of COVID-19 have been published in medical journals [4-6].

Even though most African countries have fragile health systems, the Case Fatality Rate (CFR) for COVID19 in Africa is surprisingly lower than the global trend [7]. Lower testing rates, a younger population, humid temperatures, and the possibility of a pre-existing immunity are some of the postulated factors associated with this difference [7].

Although the SARS-CoV-2 virus predominantly targets the respiratory system, its associated mortality involves multiple organ systems [8]. An increasing understanding of the disease throughout the pandemic has reduced hospital mortality rates, especially in well-resourced and high-income countries [9, $10,11]$. In contrast, hospital mortality remains comparatively high in Africa [12]. This has been attributed to the burden of underlying comorbidities and resource deficits [12]. Reports globally have indicated that increasing age $[13,14,15]$, comorbidities (cardiovascular diseases and diabetes) $[13,16,17]$, and obesity $[18,19]$ are associated with adverse outcomes. In addition, certain demographic characteristics [14-16, 20] and laboratory parameters [21-23] have also been associated with the severe form of COVID-19 and increased mortality.

The objective of this study was to identify factors associated with hospital mortality in patients who suffered from COVID-19 at Gulu Regional Referral Hospital in Northern Uganda from March 2020 to October 2021.

\section{Materials And Methods}

\section{Study design}

A retrospective data review and abstraction of all COVID-19 hospital admissions registered in the Gulu Health Management Information System (HMIS) database and other tools was conducted. The period of the review was from March 2020 to October 2021. HMIS is a database established by the Ugandan Ministry of Health as a primary source of information on COVID-19 hospital admissions and deaths. COVID-19 notification is compulsory in Uganda, and the emergency operations Centre at the Uganda National Public Health Institute receives reports on patients admitted to both public and private hospitals with COVID-19.

\section{Study site}


This study was conducted at Gulu Regional Referral Hospital in Northern Uganda, covering admissions of COVID-19 patients from March 2020 to October 2021. Gulu Hospital is a Regional and Referral Centre for patients from mid-northern Uganda., However, it receives patients from neighboring countries, for example, South Sudan and the Democratic Republic of Congo (DR Congo). It is also a teaching hospital for Gulu University Medical School and many other health training institutions in the region. It is a 394bed capacity hospital with outpatient and inpatients services estimated at 120,000 patients per year. The hospital has specialized units such as internal medicine, surgery, pediatrics, reproductive health, TB, HIV, cardiac, chest, dental, dermatology, sickle cell disease, diabetes, hypertension, Ear, Nose and throat, nutrition, accident and emergency, laboratory, ophthalmology, mental health, and orthopedic clinics that consultants manage from Gulu Hospital and Gulu University.

Gulu Regional Referral hospital was designated by the Ugandan Ministry of Health as a treatment center for COVID-19 patients in March 2020 when COVID-19 was declared a pandemic. A particular treatment unit for the management of COVID-19 (Gulu CTU) was established with a fully-fledged high dependency unit (HDU), with Oxygen supply and staff to manage the center. The COVID-19 isolation unit is housed in a separate building from other patients and comprises the general isolation ward and the COVID-19 Critical Care Unit (CCU). The CCU is split into a quasi-intensive care unit (ICU) and the high dependency unit (HDU). The general isolation ward consisted of two separate wards (one for females and another for males) with 12 beds. The COVID-19 CCU has a two-bed ICU and four-bed HDU. A multidisciplinary team of physicians, medical officers, nurses, and laboratory technicians saw the COVID-19 patients. The HDU and quasi-ICU were managed by an interdisciplinary team, including a full-time critical care specialist, primary physician, medical officers, physiotherapist, mental health specialists, and dietician. The ICU could not provide invasive procedures such as invasive mechanical ventilation, invasive hemodynamic monitoring, and inotropic support. All patients that required invasive procedures were transferred to Mulago National Referral Hospital. The HDU served as a step-down unit for the quasi-ICU and housed critically ill patients requiring high-flow oxygen. Patients requiring hemodialysis were transferred to Mulago National Referral Hospital in a separate designated dialysis unit. The ICU and HDU had round-theclock coverage with a physician, a team of medical and clinical officers from various specialties such as internal medicine and emergency medicine. The nurse-to-patient ratio for ICU and HDU was 1:1 and 1:5, respectively. The team leader for the Gulu CTU was a consultant physician who managed all the COVID19 patients admitted to the unit. Additional support for the management of the COVID-19 patients at the Centre was provided by the Ugandan Ministry of Health and World Health Organization experts using standard protocols developed and practiced in Uganda.

\section{Sources of data}

For the period of this study, COVID-19 patients admitted to Gulu Regional Referral Hospital with COVID-19 were estimated at 900. For each patient registered in the Gulu Hospital HMIS database, information on individual's socio-demographic characteristics, self-reported symptoms, signs, co-morbidities, COVID-19 Treatment Unit (CTU) admissions, HDU admissions, ICU admissions, and ventilatory support, dates of symptom onset, date of hospital admission, date of discharge, duration of hospital stay, reported 
circumstances when the disease was contracted, vaccination status and hospital outcomes (deaths, referrals, and releases/discharges) were included.

HMIS datasets were accessed, which were already de-identified and publicly available documents. Following ethically agreed principles on open data access, this review did not require stringent ethical approval in Uganda as we mainly worked on medical records with no identifiers included. However, we obtained ethical and administrative clearance from the Gulu Regional Referral Hospital Institution and Ethical Review Committee to access the archived Gulu Hospital datasets on COVID-19 patients.

\section{Selection Criteria}

\section{Inclusion criteria}

The following were the inclusion criteria for participants (i) Confirmed cases of COVID-19 with RT-PCR results as positive (ii) patients 12 years and above (iii) completed information on the chart and other medical tools (iv) admission records

\section{Exclusion criteria}

We excluded (i) incomplete records, (ii) records with no RT-PCR results (iii) participants below 12 years.

\section{Selection of records}

The medical records for the COVID-19 patients in the archives of Gulu Hospital were accessed. The choice of the COVID-19 patients' files was conducted consecutively and reviewed by the research team. The selection criteria were applied to each admission file, and a total of 664 files were included in the participating medical records for this study.

\section{Sample size}

The sample size for the study population was determined after using the selection criteria on the medical records. Six hundred and sixty-four (664) records were included as the sampled population.

\section{Training of research assistants}

To obtain excellent and clean information from the COVID-19 patients' files, the research team trained the research assistants who were four in number (Two medical officers, one clinical officer, and one nurse) on how to use the selection criteria, accurately record data from the admission forms and exclude forms that were considered incomplete. The research teams were trained on infection, prevention, and control of COVID-19 and were required to use facemasks, eye shields, and sanitizers during and after reviewing documents. The corresponding author supervised the data collection exercise from the beginning to the end, ensuring that every file was checked to confirm the completeness of the data collected.

\section{Data collection procedures}


Registered COVID-19 patients treated at Gulu Regional Referral Hospital with a positive quantitative RTPCR test result for SARS-CoV-2 admitted to Gulu Hospital were consecutively reviewed. SARS-CoV-2 diagnostic tests followed national and international standards. They were done in certified laboratories of Gulu Regional Referral Hospital and Uganda Virus Research Institute (UVRI) as required by the Ugandan Ministry of Health and World Health Organization (WHO) protocols.

\section{Variables for the study}

The dependent variables for this study were treatment outcomes (alive or dead). The independent variables were the socio-demographics of the COVID-19 patients (age, sex, occupation, religion, tribe, districts, and level of education), comorbidities and treatments used, oxygen saturation at admission, dates of discharges from the hospital, duration of hospital stay, disease severity, and others), clinical

presentations (signs and symptoms), vaccination status, residences, and circumstances under which the patient contracted the virus.

\section{Data analysis}

The analysis period was from the epidemiological week (starting month and date of March 2020) to the epidemiological week (until month and date of October 2021). The analysis was pre-specified and defined before reading the medical data in the Gulu Regional Referral Hospital records. The sample size was all patients (aged $\geq 12$ years) with COVID-19 diagnosis who were admitted and registered to the Gulu Regional Referral Hospital HMIS database between the epidemiological weeks of March 2020 to October 2021.

Means, frequencies, standard deviations, bar graphs, histograms, and percentages were used to summarize continuous variables, while frequencies and proportions were calculated for categorical variables. Age-adjusted and sex-adjusted rates for each district were calculated by the direct method using the estimated Ugandan population for 2020 as reference.

We used the Chi-Square tests to observe associations between independent and dependent variables at $95 \%$ Confidence Intervals. Factors with p-values less or equal to 0.2 were entered into a multivariable regression analysis to determine factors associated with mortality among COVID-19 patients treated at Gulu Regional Referral Hospital. However, the Gulu Hospital HMIS database contained many missing variables, such as reported symptoms, medicines used, and comorbidities. We used additional Gulu Regional Referral Hospital records to fill in the missing data. Also, in the post hoc analysis, we evaluated the missing data pattern and conducted a sensitivity analysis via multiple imputations by chained equations, generating 30 imputed datasets. All studies were performed with SPSS version 25.0. Multiple imputations were performed with SPSS following the STROBE guideline recommendations. In addition, Adjusted Odds Ratios (AoR) for independent variables associated with mortality were calculated for the COVID-19 patients treated at the Gulu Regional Referral Hospital from March 2020 to October 2021.

\section{Ethical considerations}


This retrospective data review of COVID-19 patients' medical files at the Gulu Regional Referral Hospital was approved by the Gulu Hospital Institutional, Ethics, and Review Committee.

\section{Results}

The study showed that during the period of study from March 2020, when the COVID-19 was declared a pandemic and Gulu Regional Referral Hospital became a treatment center, 32(4.8\%) COVID-19 patients died at the center. Most patients who died were females AOR=0.220, 95\%Cl:0.059-0.827; $p=0.030$; Diabetes mellitus AOR=9.014, 95\%Cl:1.726-47.067; $p=0.010$; and comorbidities for example cardiovascular diseases, hepatitis B, liver failure and HIV and AIDS AOR=6.860, 95\%Cl:1.309-35.957; $p=0.020$. Similarly, COVID-19 patients who presented with symptoms for example tiredness $A O R=0.059$, 95\%Cl:0.009-0.371; $p=0.0000$; general body aches and pains AOR=0.066, 95\%Cl:0.007-0.605; $p=0.020$, and loss of speech and movement AOR=0.134, 95\%Cl:0.270-0.660; $p=0.010$ died. Nevertheless, most COVID-19 patients treated at the Gulu Regional Referral Hospital were majorly unvaccinated $661(99.5 \%)$ against the virus and recovered from the disease at a rate of 632(95.2\%). 
Table 1

The socio-demographic characteristics of COVID-19 mortality at Gulu Regional Referral Hospital

\begin{tabular}{|c|c|c|}
\hline Variables & Frequency & Percent (\%) \\
\hline \multicolumn{3}{|l|}{ Gender } \\
\hline Male & 14 & 43.7 \\
\hline Female & 18 & 56.3 \\
\hline \multicolumn{3}{|l|}{ Age (years) } \\
\hline$<20$ & 0 & 0.0 \\
\hline $20-29$ & 3 & 9.4 \\
\hline $30-39$ & 1 & 3.1 \\
\hline $40-49$ & 9 & 28.1 \\
\hline$\geq 50$ & 19 & 59.4 \\
\hline \multicolumn{3}{|l|}{ Tribes } \\
\hline Acholi & 25 & 78.1 \\
\hline Lango & 1 & 3.1 \\
\hline Baganda & 0 & 3.1 \\
\hline Madi & 1 & 3.1 \\
\hline Others & 4 & 12.5 \\
\hline \multicolumn{3}{|l|}{ Religion } \\
\hline Catholics & 13 & 40.6 \\
\hline Protestants & 5 & 15.6 \\
\hline Born Again & 1 & 3.1 \\
\hline Muslims & 3 & 9.4 \\
\hline Others & 10 & 31.3 \\
\hline \multicolumn{3}{|c|}{ The highest level of education attained } \\
\hline No formal education & 14 & 43.0 \\
\hline Primary & 0 & 0.0 \\
\hline Secondary & 0 & 0.0 \\
\hline Certificates & 5 & 15.6 \\
\hline
\end{tabular}




\begin{tabular}{|c|c|c|}
\hline Variables & Frequency & Percent (\%) \\
\hline Diploma & 3 & 9.4 \\
\hline Degrees & 5 & 15.6 \\
\hline Postgraduate degrees & 2 & 6.3 \\
\hline \multicolumn{3}{|l|}{ Occupation } \\
\hline Business & 2 & 6.3 \\
\hline Civil Servants & 2 & 6.3 \\
\hline Health workers & 3 & 9.4 \\
\hline Teachers & 1 & 3.1 \\
\hline Uniformed security forces & 0 & 0.0 \\
\hline Peasant Farmers & 12 & 37.5 \\
\hline Others & 12 & 37.5 \\
\hline \multicolumn{3}{|l|}{ Districts } \\
\hline Agago & 1 & 3.1 \\
\hline Amuru & 2 & 6.3 \\
\hline Gulu & 15 & 46.9 \\
\hline Kitgum & 1 & 3.1 \\
\hline Lamwo & 0 & 0.0 \\
\hline Nwoya & 2 & 6.3 \\
\hline Omoro & 6 & 18.8 \\
\hline Pader & 1 & 3.1 \\
\hline Others & 4 & 12.5 \\
\hline Number of COVID-19 patients who died & 32 & 4.8 \\
\hline
\end{tabular}

Table 1 shows that most COVID-19 mortality at Gulu Regional Referral Hospital from March 2020 to October 2021 were females 18(56.3\%), age group $\geq 50$ years 19(59.4\%); no formal education $14(43.0 \%)$, Acholi 25(78.1\%), Catholics 13(40.6\%), Peasant farmers 12(37.5\%) and from Gulu District 15(46.9\%). 
Table 2

Factors associated with mortality at the bivariate analysis on the COVID-19 patients treated at Gulu Regional Referral Hospital from March 2020 to October 2021

\begin{tabular}{|c|c|c|c|c|}
\hline Variables & $\begin{array}{l}\text { Freq } \\
n=32(\%)\end{array}$ & $\begin{array}{l}\text { Chi- } \\
\text { Square }\end{array}$ & df & $\begin{array}{l}\text { p- } \\
\text { value }\end{array}$ \\
\hline Fever & $9(28.1)$ & 3.143 & 1 & 0.076 \\
\hline Cough & $25(78.1)$ & 10.639 & 1 & 0.000 \\
\hline Tiredness & $30(93.8)$ & 119.828 & 1 & 0.000 \\
\hline Body aches and pains & $31(96.9)$ & 75.543 & 1 & 0.000 \\
\hline Diarrhea & $4(12.5)$ & 10.336 & 1 & 0.001 \\
\hline Difficulty in breathing/shortness of breath/Chest pain & $32(100.0)$ & 96.929 & 1 & 0.000 \\
\hline Loss of speech and movement & $11(34.4)$ & 113.202 & 1 & 0.000 \\
\hline Headache & 19(59.4) & 9.705 & 1 & 0.002 \\
\hline Sore throat & $8(25.0)$ & 1.251 & 1 & 0.263 \\
\hline $\begin{array}{l}\text { Rashes on the skin and discoloration of toes and } \\
\text { fingers }\end{array}$ & $1(3.1)$ & 3.579 & 1 & 0.059 \\
\hline Loss of Smell & $0(0.0)$ & 2.328 & 1 & 0.127 \\
\hline Loss of Taste & $1(3.1)$ & 0.669 & 1 & 0.413 \\
\hline Diabetes Mellitus & $11(34.4)$ & 51.156 & 1 & 0.000 \\
\hline COPD & $1(3.1)$ & 1.041 & 1 & 0.307 \\
\hline Other CVDs & 12(37.5) & 34.819 & 1 & 0.000 \\
\hline Hypertension & 10(31.3) & 10.807 & 1 & 0.001 \\
\hline Obesity & $0(0)$ & 0.153 & 1 & 0.696 \\
\hline Asthma & $1(3.1)$ & 0.113 & 1 & 0.737 \\
\hline Cancer & $1(3.1)$ & 3.565 & 1 & 0.059 \\
\hline HIV and AIDS & $7(21.9)$ & 6.488 & 1 & 0.011 \\
\hline Symptomatic & $28(87.5)$ & 2.170 & 1 & 0.141 \\
\hline Oxygen saturation $\left(\mathrm{SpO}_{2}\right)(<80) 4(12.5)$ & & 62.074 & 2 & 0.0000 \\
\hline Duration of hospital stay (0-1 week) $25(78.1)$ & & 67.776 & 1 & 0.0000 \\
\hline Duration of symptoms (1-7 days) 19(59.4) & & 1.101 & 3 & 0.8970 \\
\hline
\end{tabular}

Key: $\mathrm{COPD}=$ Chronic obstructive pulmonary diseases; $\mathrm{CVDs}=$ cardiovascular diseases. 


\begin{tabular}{|c|c|c|c|c|}
\hline Variables & $\begin{array}{l}\text { Freq } \\
n=32(\%)\end{array}$ & $\begin{array}{l}\text { Chi- } \\
\text { Square }\end{array}$ & df & $\begin{array}{l}\mathrm{p}- \\
\text { value }\end{array}$ \\
\hline \multicolumn{5}{|c|}{ Systolic Blood pressure (mmHg) } \\
\hline$\leq 120$ & 13(40.6) & & & \\
\hline $121-140$ & 12(37.5) & 2.920 & 2 & 0.232 \\
\hline$>140$ & $7(21.9)$ & & & \\
\hline \multicolumn{5}{|c|}{ Diastolic Blood Pressure (mmHg) } \\
\hline$\leq 80$ & $22(68.8)$ & & & \\
\hline $81-120$ & $4(12.5)$ & 4.214 & 2 & 0.122 \\
\hline $121-140$ & $6(18.8)$ & & & \\
\hline \multicolumn{5}{|c|}{ Duration of symptoms (days) } \\
\hline $1-7$ & 19(59.4) & & & \\
\hline $8-14$ & $10(31.3)$ & 1.101 & 4 & 0.894 \\
\hline $15-21$ & $3(9.4)$ & & & \\
\hline $22-28$ & $0(0.0)$ & & & \\
\hline \multicolumn{5}{|c|}{ Ages of participants (years) } \\
\hline$<20$ & $0(0.0)$ & & & \\
\hline $20-29$ & $3(9.4)$ & & & \\
\hline $30-39$ & $1(3.1)$ & & & \\
\hline $40-49$ & $9(28.1)$ & & & \\
\hline$\geq 50$ & $19(59.4)$ & 40.601 & 4 & 0.000 \\
\hline \multicolumn{5}{|c|}{ The highest level of education attained } \\
\hline No formal education & $14(43.8)$ & 39.213 & 8 & 0.000 \\
\hline Primary level & $0(0.0)$ & & & \\
\hline Secondary level & $0(0.0)$ & & & \\
\hline Certificates & $5(15.6)$ & & & \\
\hline Diploma & $3(9.4)$ & & & \\
\hline Degrees & $5(15.6)$ & & & \\
\hline
\end{tabular}

Key: $\mathrm{COPD}=$ Chronic obstructive pulmonary diseases; $\mathrm{CVDs}=$ cardiovascular diseases. 


\begin{tabular}{|c|c|c|c|c|}
\hline Variables & $\begin{array}{l}\text { Freq } \\
n=32(\%)\end{array}$ & $\begin{array}{l}\text { Chi- } \\
\text { Square }\end{array}$ & df & $\begin{array}{l}\mathrm{p}- \\
\text { value }\end{array}$ \\
\hline Postgraduate degree & $2(6.3)$ & & & \\
\hline \multicolumn{5}{|l|}{ Tribes } \\
\hline Acholi & $25(78.1)$ & 9.511 & 7 & 0.2180 \\
\hline Langi & $0(0.0)$ & & & \\
\hline Baganda & $1(3.1)$ & & & \\
\hline Lugbara & $1(3.1)$ & & & \\
\hline Others & $4(12.5)$ & & & \\
\hline \multicolumn{5}{|l|}{ Occupation } \\
\hline Peasant Farmers & $12(37.5)$ & 33.235 & 8 & 0.0000 \\
\hline Business & $2(6.3)$ & & & \\
\hline Uniformed security & $0(0.0)$ & & & \\
\hline Civil Servants & $2(6.3)$ & & & \\
\hline Teachers & $1(3.1)$ & & & \\
\hline Health workers & $3(9.4)$ & & & \\
\hline Others & 12(37.5) & & & \\
\hline \multicolumn{5}{|l|}{ Religion } \\
\hline Born Again & $1(3.1)$ & & & \\
\hline Catholic & $13(40.6)$ & 28.691 & 5 & 0.0000 \\
\hline Moslems & $3(9.4)$ & & & \\
\hline Protestants & $5(15.6)$ & & & \\
\hline \multicolumn{5}{|l|}{ Others } \\
\hline \multicolumn{5}{|l|}{ Districts } \\
\hline Agago & $1(3.1)$ & & & \\
\hline Amuru & $2(6.3)$ & & & \\
\hline Gulu & $15(46.9)$ & 21.827 & 12 & 0.040 \\
\hline Kitgum & $1(3.1)$ & & & \\
\hline
\end{tabular}

Key: $\mathrm{COPD}=$ Chronic obstructive pulmonary diseases; $\mathrm{CVDs}=$ cardiovascular diseases. 


\begin{tabular}{|lllcc|}
\hline Variables & $\begin{array}{l}\text { Freq } \\
\mathbf{n}=32(\%)\end{array}$ & $\begin{array}{l}\text { Chi- } \\
\text { Square }\end{array}$ & $\begin{array}{c}\text { df } \\
\text { value }\end{array}$ \\
\hline Lamwo & $0(0.0)$ & & \\
\hline Nwoya & $2(6.3)$ & & & \\
\hline Omoro & $6(18.8)$ & & & \\
\hline Pader & $1(3.1)$ & & 0.002 \\
\hline Others & $4(12.5)$ & 107.107 & & \\
\hline Gender & & & & 0.005 \\
\hline Females & $18(56.3)$ & 7.986 & & \\
\hline Males & $14(43.7)$ & & & \\
\hline Key: COPD= Chronic obstructive pulmonary diseases; CVDs=cardiovascular diseases. & & \\
\hline
\end{tabular}

In Table 2, factors associated with mortality at bivariate analysis were cough $\chi^{2}=10.639 ; p=0.000$; Tiredness $\chi^{2}=6.488 ; p=0.000$; Age $\left(\geq 50\right.$ years) $\chi^{2}=40.601 ; p=0.000$; No formal education $\chi^{2}=39.213$; $p=0.000$; Peasant Farmer $\chi^{2}=\chi^{2}=119.828 ; p=0.000$; Body aches and pains $\chi^{2}=75.543 ; p=0.000$; Diarrhoea $\chi^{2}=10.336 ; p=0.001$; Difficulty in breathing/shortness of breath/Chest pain $\chi^{2}=96.929$; $p=0.000$; Loss of speech and movement $\chi^{2}=113.202 ; p=0.001$; Headache $\chi^{2}=9.705 ; p=0.002$; Diabetes Mellitus $\chi^{2}=51.156 ; p=0.000$; Other CVDs $\chi^{2}=34.819 ; p=0.000$; Hypertension $\chi^{2}=10.807 ; p=0.000 ;$ HIV and AIDS $\chi^{2}=6.488 ; p=0.011$; Oxygen saturation $\left.\left(\mathrm{SpO}_{2}\right)<80\right) \chi^{2}=62.074 ; \mathrm{p}=0.000$; Duration of Hospital stay (01 week) 33.235; $p=0.000$; Catholics $\chi^{2}=28.691 ; p=0.000 ;$ Gulu district $\chi^{2}=21.827 ; p=0.040$ and Females $\chi^{2}=7.986 ; p=0.005$ 
Table 3

The adjusted Odds Ratios for factors associated with mortality among COVID-19 patients treated at Gulu Regional Referral Hospital

\begin{tabular}{|c|c|c|c|c|c|c|c|c|}
\hline \multirow[t]{2}{*}{ Variables } & \multirow[t]{2}{*}{ B } & \multirow[t]{2}{*}{ S.E. } & \multirow[t]{2}{*}{ Wald } & \multirow[t]{2}{*}{ df } & \multirow[t]{2}{*}{ Sig. } & \multirow[t]{2}{*}{$\operatorname{Exp}(B)$} & \multicolumn{2}{|c|}{$\begin{array}{l}95 \% \text { C.I. for } \\
\text { EXP(B) }\end{array}$} \\
\hline & & & & & & & Lower & Upper \\
\hline Symptomatic (1) & 0.749 & 0.792 & 0.893 & 1 & 0.345 & 2.114 & 0.448 & 9.990 \\
\hline Sex (Female) & -1.514 & 0.676 & 5.025 & 1 & 0.030 & 0.220 & 0.059 & 0.827 \\
\hline $\begin{array}{l}\text { Diabetes Mellitus } \\
\text { (1) }\end{array}$ & 2.199 & 0.843 & 6.799 & 1 & 0.010 & 9.014 & 1.726 & 47.067 \\
\hline $\begin{array}{l}\text { Chronic obstructive } \\
\text { pulmonary disease } \\
\text { (COPD)(1) }\end{array}$ & -0.818 & 1.69 & 0.234 & 1 & 0.628 & 0.441 & 0.016 & 12.106 \\
\hline $\begin{array}{l}\text { Other } \\
\text { Cardiovascular } \\
\text { Diseases (CVD) (1) }\end{array}$ & 1.953 & 1.874 & 1.086 & 1 & 0.297 & 7.050 & 0.179 & 277.547 \\
\hline Fever (1) & -0.172 & 0.72 & 0.057 & 1 & 0.811 & 0.842 & 0.205 & 3.454 \\
\hline Cough (1) & 1.220 & 0.885 & 1.898 & 1 & 0.168 & 3.386 & 0.597 & 19.192 \\
\hline Tiredness (1) & -2.835 & 0.941 & 9.078 & 1 & 0.000 & 0.059 & 0.009 & 0.371 \\
\hline Aches and pains (1) & -2.714 & 1.128 & 5.786 & 1 & 0.020 & 0.066 & 0.007 & 0.605 \\
\hline Sore throat (1) & -0.737 & 0.748 & 0.972 & 1 & 0.324 & 0.478 & 0.110 & 2.073 \\
\hline Vomiting (1) & 0.189 & 1.068 & 0.031 & 1 & 0.859 & 1.209 & 0.149 & 9.804 \\
\hline Diarrhea (1) & 1.153 & 1.109 & 1.080 & 1 & 0.299 & 3.167 & 0.360 & 27.853 \\
\hline Conjunctivitis (1) & 14.818 & 40193 & 0.000 & 1 & 1.0000 & 2725229 & 0.000 & . \\
\hline $\begin{array}{l}\text { Loss of speech or } \\
\text { movement (1) }\end{array}$ & -2.008 & 0.812 & 6.112 & 1 & 0.010 & 0.134 & 0.027 & 0.660 \\
\hline $\begin{array}{l}\text { Hypertension (HT) } \\
\text { (1) }\end{array}$ & -1.273 & 1.913 & 0.443 & 1 & 0.506 & 0.2800 & 0.007 & 11.886 \\
\hline Obesity (1) & 0.033 & 45730 & 0.000 & 1 & 1.0000 & 1.0330 & 0.000 & . \\
\hline Asthma (1) & -0.870 & 1.651 & 0.277 & 1 & 0.598 & 0.4190 & 0.016 & 10.664 \\
\hline Cancer (1) & 0.379 & 2.152 & 0.031 & 1 & 0.860 & 1.4610 & 0.022 & 99.174 \\
\hline HIV (1) & 1.112 & 0.785 & 2.007 & 1 & 0.157 & 3.0410 & 0.653 & 14.166 \\
\hline $\begin{array}{l}\text { Other comorbidities } \\
\text { (1) }\end{array}$ & 1.926 & 0.845 & 5.191 & 1 & 0.020 & 6.860 & 1.309 & 35.957 \\
\hline Age (years) & & & & & & & & \\
\hline
\end{tabular}




\begin{tabular}{|c|c|c|c|c|c|c|c|c|}
\hline \multirow[t]{2}{*}{ Variables } & \multirow[t]{2}{*}{ B } & \multirow[t]{2}{*}{ S.E. } & \multirow[t]{2}{*}{ Wald } & \multirow[t]{2}{*}{ df } & \multirow[t]{2}{*}{ Sig. } & \multirow[t]{2}{*}{$\operatorname{Exp}(B)$} & \multicolumn{2}{|c|}{$\begin{array}{l}95 \% \text { C.l. for } \\
\text { EXP(B) }\end{array}$} \\
\hline & & & & & & & Lower & Upper \\
\hline$<20$ years & -17.04 & 8584.01 & 0.000 & 1 & 0.998 & 0.0000 & 0.0000 & . \\
\hline 20-29 years & -15.37 & 8584.01 & 0.000 & 1 & 0.999 & 0.0000 & 0.0000 & . \\
\hline $30-39$ years & -18.53 & 8584.01 & 0.000 & 1 & 0.998 & 0.0000 & 0.0000 & . \\
\hline $40-49$ years & -19.39 & 8584.01 & 0.000 & 1 & 0.998 & 0.0000 & 0.0000 & . \\
\hline $\begin{array}{l}\text { Duration of } \\
\text { Symptoms }\end{array}$ & & & 0.121 & 4 & 0.998 & & & \\
\hline 1-7 days & -0.036 & 0.621 & 0.003 & 1 & 0.954 & 0.965 & 0.286 & 3.26 \\
\hline 8-14 days & 0.352 & 1.112 & 0.100 & 1 & 0.751 & 1.422 & 0.161 & 12.564 \\
\hline 15-21 days & 18.985 & 21812.1 & 0.000 & 1 & 0.999 & $1.8 \mathrm{E}+08$ & 0.0000 & . \\
\hline $22-28$ days & 18.553 & 27748.4 & 0.000 & 1 & 0.999 & $1.1 \mathrm{E}+08$ & 0.0000 & . \\
\hline $\begin{array}{l}\text { The highest level of } \\
\text { education attained }\end{array}$ & & & 4.790 & 8 & 0.780 & & & \\
\hline $\begin{array}{l}\text { No formal } \\
\text { education }\end{array}$ & 19.173 & 7639.02 & 0.000 & 1 & 0.998 & $2.1 \mathrm{E}+08$ & 0.0000 & . \\
\hline Primary level & 18.541 & 6963.22 & 0.000 & 1 & 0.998 & $1.1 \mathrm{E}+08$ & 0.0000 & . \\
\hline Secondary level & 0.455 & 0.773 & 0.347 & 1 & 0.556 & 1.576 & 0.3470 & 7.166 \\
\hline Certificate & 1.547 & 1.053 & 2.157 & 1 & 0.142 & 4.698 & 0.5960 & 37.023 \\
\hline Diploma & 1.557 & 0.895 & 3.026 & 1 & 0.080 & 4.746 & 0.8210 & 27.44 \\
\hline Degree & 1.797 & 1.172 & 2.351 & 1 & 0.125 & 6.029 & 0.6070 & 59.93 \\
\hline Postgraduate & 20.64 & 40193 & 0.000 & 1 & 1.000 & $9.2 \mathrm{E}+08$ & 0.0000 & \\
\hline Constant & 15.97 & 46528.6 & 0.000 & 1 & 1.000 & 8623109 & & \\
\hline
\end{tabular}

In Table 3, the Adjusted Odds Ratios (AOR) of factors associated with mortality among COVID-19 patients treated at Gulu Regional Referral Hospital were; Females AOR=0.220, 95\%Cl:0.059-0.827; $p=0.030$; Diabetes mellitus AOR=9.014, 95\%Cl:1.726-47.067; $p=0.010$; Tiredness AOR=0.059, 95\%Cl:0.009-0.371; $p=0.0000$; Body aches and pains AOR $=0.066,95 \% \mathrm{Cl}: 0.007-0.605 ; p=0.020$; Loss of speech and movement AOR=0.134, 95\%Cl:0.270-0.660; $p=0.010$ and other comorbidities $A O R=6.860,95 \% \mathrm{Cl}: 1.309-35.957$; $p=0.020$. 
Table 4

Factors associated with female gender among the COVID-19 patients

Variables

Chi-square

df

value

Crosstabulations between duration of symptoms (days) and other variables

Symptomatic patients

10.301

$4 \quad 0.036$

Age of patients

14.585

$16 \quad 0.555$

Female patients

6.284

$4 \quad 0.179$

The highest level of education attained

30.42

$32 \quad 0.547$

Crosstabulations between Diabetes Mellitus and other variables

Symptomatic patients

5.314

$1 \quad \mathbf{0 . 0 2 1}$

Age of patients

22.66

$4 \quad 0.000$

Female patients

0.016

$1 \quad 0.901$

The highest level of education attained

32.532

$8 \quad 0.000$

Crosstabulations between Chronic obstructive pulmonary diseases (COPDs) and other variables

Symptomatic patients

0.014

10.905

Age of patients

6.195

4

0.185

Female patients

6.346

1

0.032

The highest level of education attained

Crosstabulations between Other cardiovascular diseases (CVDs) and other variables

Symptomatic patients

Age of the patients

Female patients

The highest level of education attained

Crosstabulations between Hypertension and other variables

Symptomatic patients

Age of the patients

Female patients

The highest level of education attained

Crosstabulations between obesity and other variables
4.462

22.562

4.996

22.451

3.045

$1 \quad 0.081$

35.169

$4 \quad 0.000$

1.187

1

0.276

21.624

$8 \quad 0.006$ 


\begin{tabular}{|llll|}
\hline Variables & Chi-square & df & $\begin{array}{c}\text { p- } \\
\text { value }\end{array}$ \\
\hline Symptomatic patients & 0.173 & 1 & 0.678 \\
\hline Age of patients & 1.272 & 4 & 0.866 \\
\hline Female patients & 1.512 & 1 & 0.219 \\
\hline The highest level of education attained & 2.083 & 8 & 0.978 \\
\hline Crosstabulations between Asthma and other variables & & & \\
\hline Symptomatic patients & 0.0920 & 1 & 0.762 \\
\hline Age of patients & 3.7700 & 4 & 0.438 \\
\hline Female patients & 2.8110 & 1 & 0.094 \\
\hline The highest level of education attained & 10.3790 & 8 & 0.239 \\
\hline Crosstabulations between Cancer and other variables & & & \\
\hline Symptomatic patients & 1.2200 & 1 & 0.269 \\
\hline Age of patients & 1.7470 & 4 & 0.782 \\
\hline Female patients & 3.1440 & 1 & 0.076 \\
\hline The highest level of education attained & 12.6480 & 8 & 0.125 \\
\hline Crosstabulations between HIV and AIDS and other variables & & 8 & 0.978 \\
\hline Symptomatic patients & 0.070 & 1 & 0.791 \\
\hline Age of patients & 8.515 & 4 & 0.074 \\
\hline Female patients & 3.646 & 1 & 0.056 \\
\hline The highest level of education attained & 2.107 & 8 & \\
\hline Table & & & \\
\hline
\end{tabular}

In Table 4, the female COVID-19 patients were statistically and significantly associated with cardiovascular diseases $\left(\chi^{2}=4.996 ; p=0.025\right)$ and Chronic Obstructive Pulmonary Diseases (COPDs) $\chi^{2}=$ 6.346; $p=0.032$ and with close to significant associations with HIV and $\operatorname{AIDS}\left(\chi^{2}=3.646 ; p=0.056\right)$ and Cancers $\left.\chi^{2}=3.144 ; p=0.076\right)$.

\section{Discussions}

The mortality rate among COVID-19 patients treated at Gulu Regional Referral Hospital was 4.8\% (Table 1). This mortality rate was much lower than the average mortality rates reported in Africa $[6,12,24]$ but slightly higher than the global average amongst hospitalized patients at $3.8 \%[6,24]$. Authors suggest the low mortality rates were attributable to the mild nature of the COVID-19 disease and relatively fewer patients admitted to the Gulu Regional Referral Hospital with comorbidities. This was shown by the 
clinical presentations where most patients had oxygen saturation $\left(\mathrm{SpO}_{2}\right)$ more than 96 , and one-fourth of the patients were asymptomatic. At the same time, symptomatic cases were mild, and most patients presented early in the course of the disease (Table 2).

The study identified several risk factors associated with mortality among hospitalized patients with COVID-19 in the Ugandan setting. Our study found females, Diabetes mellitus, comorbidities (cardiovascular diseases, hepatitis B, HIV and AIDS, Chronic Obstructive pulmonary diseases (COPDs), liver failure, and severe malaria), and severe illnesses as shown by symptoms such as tiredness, general body aches, and pains, and loss of speech and movements as the main factors associated with higher odds of mortality (Table 3). This was similarly observed in other studies $[6,13,16,17]$.

Furthermore, this study found a higher chance of death in COVID-19 patients with diabetes mellitus (Table 3). Studies found that hyperglycemia secondary to Diabetes mellitus leads to immune dysfunction by impairing humoral and cellular functions and the antioxidant system [25, 26]. In addition, studies showed that diabetic patients were more susceptible to nosocomial infections [25, 26]. These factors may have contributed to the higher chances of death in diabetic patients with COVID-19, which we observed in our study population.

Although the age group of 50 years and above 19(59.4\%) were the majority among those who died, it did not reach statistical significance in this current study (Table 2). Previous studies had shown that adults were more likely to suffer from severe forms of the disease $[8,27,28]$. In many studies, the median age of hospitalized patients with COVID-19 ranged from 49 to 56 years $[8,27,28]$. This was consistent with reports published globally and comparable to other African studies [12, 29].

Furthermore, these authors argue that although the older age group in our study did not reach statistical significance between age and increased odds of mortality, the association between the older age group with higher levels of angiotensin-converting enzyme 2 (ACE2), which is a cell surface receptor for SARSCoV-2 were plausible explanations [30].

Studies have shown that the elderly and males were at increased mortality risks $[8,27,28]$. This contrasts with our study, where middle-aged persons and females were the most affected (Table 3 ). This information requires further explanation as females in Northern Uganda suffered more mortalities from COVID-19 than males (Table 1 and Table 3), yet more males were admitted with the disease. Authors have found that the female gender in Northern Uganda had statistically significant associations with cardiovascular diseases $\left(\chi^{2}=4.996 ; p=0.025\right)$ and chronic obstructive pulmonary diseases (COPDs) $\chi^{2}=$ 6.346; $p=0.032$, with close significant associations with HIV and AIDS $\left(\chi^{2}=3.646 ; p=0.056\right)$ and Cancers $\left.\chi^{2}=3.144 ; p=0.076\right)$. Cardiovascular diseases are comorbidities that lead to severe illness, hospitalization, and death from the COVID-19 $[13,16,17]$. These authors further argue that the high prevalence of HIV and AIDS and cancers among females in Northern Uganda may have contributed to the high mortality rates observed in the current study. 
Recent studies from Northern Uganda showed a higher prevalence of HIV and AIDS among female gender in Northern Uganda at 17\% compared to males at 9\% [31]. Similarly, most cancer prevalence was higher in Northern Uganda than in the rest of the country, especially breast and cervical cancers [32]. These authors argue that these three factors (cardiovascular diseases, chronic obstructive pulmonary diseases (COPDs), HIV and AIDS, and Cancers) may have singly or collectively contributed to the increased mortality risks of COVD-19 among females in Northern Uganda.

In addition, tiredness, general body aches, body pains, headache, loss of speech and movement, vomiting, dyspnea, and cough were the symptoms found in the COVID-19 mortality at Gulu Regional Referral Hospital. This is supported by studies that showed that these symptoms and signs were more frequent in SARS cases and cases of death [27-29, 33].

Dyspnea is the main symptom of SARS, a severe form of the COVID-19 disease where more chances of death occur [33]. At the same time, other reported symptoms, such as headache, general body aches, body pains, diarrhea, loss of speech and movements, and tiredness, were associated with higher chances of death among this cohort (Table 2 and Table 3 ). However, loss of smell and taste, sore throat, rashes on the skin, toes, nails, and runny nose were not associated with death in this current study (Table 2 and Table 3). This contrasts with Patrícia Rezende do Prado et al., which reported dyspnea as a factor related to COVID-19 death, while cough, fever, and other symptoms were protective factors [33].

Taking these results, it can be concluded that this study analyzed the epidemiological characteristics and mortality risk factors in individuals diagnosed with COVID-19 due to SARS-CoV-2 at Gulu Regional Referral Hospital in Northern Uganda. High-risk groups need special attention, especially the elderly and those with comorbidities such as diabetes mellitus, cancers, HIV and AIDS, cardiovascular diseases, hepatitis $B$, and liver failure. Particular attention should be accorded to patients with dyspnea, general body pains and aches, loss of speech and movement, tiredness, headache, vomiting, and diarrhea. Some symptoms were more frequent in mild cases of COVID-19 and should be better elucidated in future studies. The female gender had more mortality risks in this current study due to comorbidities (cardiovascular diseases, chronic obstructive pulmonary diseases (COPDs), HIV and AIDS, and cancers) (Table 4). It is proposed that special care be accorded to females who contract COVID-19 to screen out for comorbidities in the early management phases so that the morbidity and mortality are averted.

\section{Strengths and limitations of this study}

This study was a retrospective review of datasets from the COVID-19 medical records at Gulu Regional Referral Hospital from March 2020 to October 2021. The study has limitations on how the Gulu Regional Referral Hospital handled records and record keeping. In addition, vital information, such as weight, height, and BMI of COVID-19 patients, was not recorded due to the emergency handling of the cases at the beginning of the pandemic in March 2020. The missing variables in the Hospital HMIS records got some files excluded from the participating records for this study. In this, authors have suggested a need for a prospective or longitudinal assessment of the COVID-19 cases in the future, ensuring that all data were measured and recorded appropriately. 
This data is vital as it is one of the well-documented completed data for over 664 cases of COVID-19 treated in a Regional Referral Hospital in Uganda. Findings from this study show tremendous and good clinical practices at the Gulu Regional Referral Hospital despite the logistical challenges faced during the pandemic.

\section{Generalizability of the results}

These findings should be cautiously interpreted and generalized to regional hospital findings in lowresource settings such as Uganda.

\section{Conclusion}

\section{Conclusion}

The overall hospital mortality was $4.8 \%$. Older age groups, diabetics, females, and those with comorbidities, severe forms of the disease, and admitted to HDU were significant risk factors associated with hospital mortality. More efforts should be made to shield the most vulnerable from the public from COVID-19 to avoid preventable morbidity and mortality in Northern Uganda.

\section{Declarations}

Ethics approval and consent to participate: The Gulu University IREC approved this study, and all participants consented to the study. In addition, the study was conducted following the relevant institutional guidelines and regulations.

Consent for publication: All participants consented to publication information from this study.

Availability of data and material: All datasets supporting the conclusion in this article is within this article and is accessible by a reasonable request to the corresponding author.

Competing interests: All authors declare no conflict of interest.

Funding: Most funding for this study was contributions of individual research members of the Uganda Medical Association (UMA) Acholi branch.

Authors' contributions: DLK, ENI, PL, JNO, JA, JO, and FWDO participated in designing the study, SB and DLK were responsible for data abstraction supervision, ENI, and DLK were responsible for data analysis, interpretation, BS, CO, NAO, WAO, BT, JE, PA, PL, DA, JNO, DO, POO, OS, FWDO, JA, DLK for writing and revising the manuscript.

Acknowledgment: We acknowledge with many thanks to the support from the administration of Gulu Regional Referral Hospital for the information obtained. We recognize Dr. Laban Oketcho, Dr. Baguma Steven, Dr. Anek Janet Schola, and Mr. Dominic Ogwal Savio for the data well collected. The 
comprehensive data analysis was conducted by Mr. Lawrence Oketayot Oballim. Financial support from UMA Acholi branch members, which enabled the team to conduct this study successfully, is most appreciated. In honor of our two fallen colleagues (Co-authors), Dr. Paska Layet and Dr. Oola Janet, may their souls rest in peace!

\section{References}

1. Phelan AL, Katz R, Gostin LO. The novel coronavirus originating in Wuhan, China: challenges for global health governance. JAMA. 2020;323(8):709-710. doi:10.1001/jama.2020.1097

2. Alimohamadi $Y$, Taghdir M, Sepandi M. The Estimate of the Basic Reproduction Number for Novel Coronavirus disease (COVID-19): A Systematic Review and Meta-Analysis. J Prev Med Public Health. 2020; 53(3):151-157.

3. Bonilla-Aldana DK, Dhama K, Rodriguez Morales AJ. Revisiting the one health approach in the context of COVID-19: an Iran J Public Health. 2020;49(7):1211-1221

4. Anderson RM, Heesterbeek $\mathrm{H}$, Klinkenberg $\mathrm{D}$, et al. How will country-based mitigation measures influence the course of the COVID-19 epidemic? Lancet. 2020;395(10228):931-4

5. Guo Y-R, Cao Q-D, Hong Z-S, et al. The origin, transmission, and clinical therapies on coronavirus disease 2019 (COVID-19) outbreak-an update on the status. Mil Med Res. 2020;7(1):11

6. World Health Organization (WHO). The WHOChina Joint Mission on Coronavirus Disease 2019 (COVID-19) report. 2020. Available from: https://www.who.int/docs/default source/coronavirus/whochina-joint-mission-on-covid-19-final-report.pdf

7. Njenga MK, Dawa J, Nanyingi M, et al. Why is There Low Morbidity and Mortality of COVID-19 in Africa? Am J Trop Med Hyg. 2020;103(2):564-569. doi:10.4269/ajtmh.20-0474

8. Huang C, Wang Y, Li X, et al. Clinical features of patients infected with 2019 novel coronavirus in Wuhan, China. Lancet. 2020;395 (10223):497-506. doi:10.1016/S0140-6736(20)30183-5

9. Dennis JM, McGovern AP, Vollmer SJ, Mateen BA. Improving Survival of Critical Care Patients with Coronavirus Disease 2019 in England: a National Cohort Study, March to June 2020. Crit Care Med. 2021;49(2):209-214. doi:10.1097/CCM.0000000000004747

10. Horwitz LI, Jones SA, Cerfolio RJ, et al. Trends in COVID-19 Risk-Adjusted Mortality Rates. J Hosp Med. 2021;16(2):90-92. doi:10.12788/jhm.3552

11. Anesi GL, Jablonski J, Harhay MO, et al. Characteristics, Outcomes, and Trends of Patients with COVID-19-Related Critical Illness at a Learning Health System in the United States. Ann Intern Med. 2021;174(5):613-621. doi:10.7326/M20-5327

12. African C. Patient care and clinical outcomes for patients with COVID-19 infection admitted to African high-care or intensive care units (ACCCOS): a multicentre, prospective, observational cohort study. Lancet. 2021;397 (10288):1885-1894. doi:10.1016/S0140-6736(21)00441-4

13. Wu Z, McGoogan JM. Characteristics of and Important Lessons from the Coronavirus Disease 2019 (COVID-19) Outbreak in China: summary of 72314 cases reported from the Chinese Center for 
Disease Control and Prevention. JAMA. 2020;323 (13):1239-1242. doi:10.1001/jama.2020.2648

14. Richardson S, Hirsch JS, Narasimhan M, et al. Presenting Characteristics, Comorbidities, and Outcomes Among 5700 Patients Hospitalized With COVID-19 in the New York City Area. JAMA. 2020;323(20):2052-2059. doi:10.1001/jama.2020.6775

15. Onder G, Rezza G, Brusaferro S. Case-Fatality Rate and Characteristics of Patients Dying about COVID-19 in Italy. JAMA. 2020;323(18):1775-1776

16. Petrilli CM, Jones SA, Yang J, et al. Factors associated with hospital admission and critical illness among 5279 people with coronavirus disease 2019 in New York City: prospective cohort study. BMJ. 2020;369:m1966. doi:10.1136/bmj.m1966

17. Williamson EJ, Walker AJ, Bhaskaran K, et al. Factors associated with COVID-19-related death using OpenSAFELY. Nature. 2020;584 (7821):430-436. doi:10.1038/s41586-020-2521-4

18. Lighter J, Phillips M, Hochman S, et al. Obesity in Patients Younger Than 60 Years Is a Risk Factor for COVID-19 Hospital Admission. Clin Infect Dis. 2020;71(15):896-897. doi:10.1093/cid/ciaa415

19. Tartof SY, Qian L, Hong V, et al. Obesity and Mortality Among Patients Diagnosed With COVID-19: results From an Integrated Health Care Organization. Ann Intern Med. 2020;173(10):773-781. doi:10.7326/M20-3742

20. Chen $T$, Wu D, Chen $H$, et al. Clinical characteristics of 113 deceased patients with coronavirus disease 2019: a retrospective study. BMJ. 2020;368:m1091. doi:10.1136/bmj.m1091

21. Zhou F, Yu T, Du R, et al. Clinical course, and risk factors for mortality of adult inpatients with COVID19 in Wuhan, China: a retrospective cohort study. Lancet. 2020;395(10229):1054-1062. doi:10.1016/S0140-6736(20)30566-3

22. Wu C, Chen X, Cai Y, et al. Risk Factors Associated with Acute Respiratory Distress Syndrome and Death in Patients with Coronavirus Disease 2019 Pneumonia in Wuhan, China. JAMA Intern Med. 2020;180(7):934-943. doi:10.1001/jamainternmed.2020.0994

23. Shi S, Qin M, Shen B, et al. Association of Cardiac Injury with Mortality in Hospitalized Patients With COVID-19 in Wuhan, China. JAMA Cardiol. 2020;5(7):802-810. doi:10.1001/jamacardio.2020.0950

24. Abate SM, Check YA, Mantefardo B. Global prevalence, and determinants of mortality among patients with COVID-19: a systematic review and meta-analysis. Ann Med Surg (Lond).2021;64:102204. doi:10.1016/j.amsu.2021.102204

25. Klonoff DC, Umpierrez GE. Letter to the editor: COVID-19 in patients with diabetes: risk factors that increase morbidity. Metabolism. 2020;108:154224 Available from:

https://doi.org/10.1016/j.metabol.2020.154224.

26. Marcella Cini Oliveira, Tatiana de Araujo Eleuterio, Allan Bruno de Andrade Corrêa, Lucas Dalsenter Romano da Silva, Renata Coelho Rodrigues, Bruna Andrade de Oliveira, Marlos Melo Martins, Carlos Eduardo Raymundo and Roberto de Andrade Medronh. Factors associated with death in confirmed cases of COVID-19 in the state of Rio de Janeiro. BMC Infectious Diseases.2021;21:687 https://doi.org/10.1186/s12879-021-06384-1 
27. Chen N, Zhou M, Dong X, et al. Epidemiological and clinical characteristics of 99 cases of 2019 novel coronavirus pneumonia in Wuhan, China: a descriptive study. Lancet. 2020;395 (10223):507-513. doi:10.1016/S0140-6736(20)30211-7

28. 28. Wang D, Hu B, Hu C, et al. Clinical Characteristics of 138 Hospitalized Patients With 2019 Novel Coronavirus-Infected Pneumonia in Wuhan, China. JAMA. 2020;323(11):1061-1069. doi:10.1001/jama.2020.1585

29. 29. Abraha HE, Gessesse Z, Gebrecherkos T, et al. Clinical features and risk factors associated with morbidity and mortality among patients with COVID-19 in northern Ethiopia. Int J Infect Dis. 2021;105:776-783. doi:10.1016/j.ijid.2021.03.037

30. 30. Wallentin L, Lindback J, Eriksson N, et al. Angiotensin-converting enzyme 2 (ACE2) levels about risk factors for COVID-19 in two large cohorts of patients with atrial fibrillation. Eur Heart J. 2020;41(41):4037-4046. doi:10.1093/eurheartj/ehaa697

31. Mary Assumpta Aduk, Isaac Malon Atama, Leonard Dayo, Cissy Namujuzi Kizza, Brian Musinguzi, Pretty Precious Ndomeirwe, William Okiror, Ignatius Senteza, Grace Wachira Wanjiku, Eric Nzirakaindi Ikoona, David Lagoro Kitara. Voluntary HIV Counseling and Testing (VCT): Knowledge, attitudes, hindrances, and practices of adults seeking treatment at Gulu Regional Referral Hospital on the spread of HIV in Northern Uganda. A cross-sectional study. Research Square. 2021. DOI: https://doi.org/10.21203/rs.3.rs-1173983/v1

32. Joyce Ayugi, George Ndagijimana, Stanley Luyima, David Lagoro Kitara. Breast cancer awareness and downstaging practices among adult women in the Gulu City Main Market, Northern Uganda: A cross-sectional study. Research Square. 2021. DOl: https://doi.org/10.21203/rs.3.rs-1136303/v1

33. Patrícia Rezende do Prado, Fernanda Raphael Escobar Gimenes, Marcos Venicius Malveira de Lima, Virgilio Batista do Prado, Carolina Pontes Soares, Thatiana Lameira Maciel Amaral. International Journal of General Medicine. 2021;14:5431-5440

\section{Supplementary Files}

This is a list of supplementary files associated with this preprint. Click to download.

- LogisticRegression1.docx 